\title{
Special Issue: Statistical mathematics for ecological and environmental data
}

\author{
Ichiro Ken Shimatani ${ }^{1} \cdot$ Kunio Shimizu ${ }^{2}$
}

Accepted: 6 November 2020 / Published online: 17 November 2020

(c) Springer Science+Business Media, LLC, part of Springer Nature 2020

On March 25 and 26, 2019, the annual Symposium on Environmental Statistics took place at The Institute of Statistical Mathematics (ISM), Tokyo, Japan. This series of international symposia started in 2013 and has continued since then, including a joint symposium with The Australian National University and University of Canberra in 2016. The 2020 symposium was cancelled due to the COVID-19 pandemic. Each symposium is comprised of a variety of presentations ranging from mathematical statistics to more applied, environmental studies. This interdisciplinarity originates from the Akaike era (Hirotugu Akaike, 1927-2009), as the Akaike Information Criterion (AIC) and related statistical ideas were born within the walls of the ISM. As indicated by the name, research conducted at ISM aims at statistical mathematics, instead of mathematical statistics. A program of the symposia of the past seven years can be found at https://www.ism.ac.jp/events/2019/meeting0325_26.html.

The 75th anniversary of the launching of the ISM was celebrated in 2019, and that year, 10 invited speakers from six countries, together with commentators and general audience, discussed various issues in ecological and environmental statistics at the annual symposium. Datasets ranged from species diversity throughout Japan and individual tree distribution in forests, to PM2.5 concentration, gaseous elemental mercury, earthquakes, climate, insect pest damage, and fishery. Spatial and spatio-temporal statistical models ranged from geostatistical to point processes, cluster analysis, uncertainty visualization, Bayesian regression with jumps, and hierarchies.

This EEST special issue includes four papers. First, Nowak and Welsh developed spatio-temporal predictions based on correlations between training and new data, showed computational efficiency if reduced subsets of data are used, depending on whether the framework for prediction is temporal, spatial or spatio-temporal, and applied their method to rainfall data. Second, after a review of previous approaches to

\footnotetext{
$凶 \quad$ Ichiro Ken Shimatani shimatan@ism.ac.jp

Kunio Shimizu

k-shmz@ism.ac.jp

1 The Risk Analysis Research Center, The Institute of Statistical Mathematics, Tachikawa, Japan

2 School of Statistical Thinking, The Institute of Statistical Mathematics, Tachikawa, Japan
} 
anisotropic covariogram modelling in spatial statistics, Koch, Lele and Lewis proposed an extension of a separable anisotropic covariance function, showed its flexibility in applications and ability to capture anisotropic characteristics of spatial data even under model misspecification, and demonstrated these properties on mountain pine beetle damage data. Third, some fishery vessels are believed to cooperate with each other and their trajectories should be related to the abundance of the target fish. Applying an appropriate cluster analysis technique to vessel location data, Lennert-Cody et al. quantitatively characterized cooperative fishery activities. Fourth, earthquake datasets can be interpreted as realizations of a 2-D spatial and temporal point process with two marks (magnitude, hypocenter depth), or depth can provide a third spatial dimension. Searching for periodicities at the monthly scale, Dutilleul, Johnson and Bürgmann apply statistical methods for temporal series analysis (e.g., multi-frequential periodogram and derived coherency), with original earthquake data as well as 2-D and 3-D declustered earthquake data, and discuss the differences between results.

We acknowledge with thanks the encouragements and support from the Network of Excellence (NOE) project in the Risk Analysis Research Center at the ISM, and the past Director-General Dr. Higuchi, the present Director-General Dr. Tsubaki, the Vice Director-Generals Dr. Ito and Dr. Yamashita at the ISM. During the course of the symposium series, we deeply appreciated the efforts and cooperation of Dr. Kanefuji, Dr. Welsh, Dr. Rivest, Dr. Liu, Dr. Chen, and Dr. Dutilleul who also sent the invitation for a special issue to be published in EEST. Finally, we want to express our gratitude to all the speakers in the 2019 symposium and the authors and reviewers of the papers. 